\title{
Tackling the worldwide loss of leprosy expertise
}

\author{
Geoff Warne ${ }^{\mathrm{a}} \&$ Monty Mukhier ${ }^{\mathrm{a}}$ \\ anternational Federation of Anti-Leprosy Associations (ILEP), Route du \\ Nant-d'avril 150, 1217 Meyrin, Switzerland
}

Submitted and accepted 7 May 2021

\begin{abstract}
Summary Limited or dwindling leprosy expertise at all levels is a challenge in almost every leprosy-endemic country and is a major obstacle to achieving zero leprosy. This report describes key findings from a 2020 conference of ILEP Member associations which examined the issue from multiple perspectives. The consensus was that continuing to use the traditional training tools and approaches is unlikely to improve the situation: innovative approaches are needed. The report focuses on the dimensions of the problem, what people need to know at different levels, learning design and pedagogy, and modes of delivery of effective and sustainable training especially using digital technologies. A number of action steps are suggested for ILEP and partners.
\end{abstract}

Keywords: Training, practice-theory, knowledge

\section{Introduction}

Limited or dwindling leprosy capacity or expertise is listed in the WHO Global Leprosy Strategy 2021-2030 as one of the major challenges on the path towards zero leprosy, defined as zero transmission, zero disability and zero stigma and discrimination. ${ }^{1}$ Capacity is an issue in almost every endemic country, at all levels in the health workforce, and in all aspects of leprosy prevention, screening, diagnosis, treatment, programme management and monitoring and evaluation.

The International Federation of Anti-Leprosy Associations (ILEP) holds periodic conferences to enable Member associations to work collaboratively on matters of mutual interest and concern. The September 2020 conference tackled the challenges arising from the erosion of leprosy expertise worldwide. 165 people participated in this virtual conference, more than 90 of whom were from leprosy-endemic countries. There were presentations from ten organisations on innovative approaches to the challenges around leprosy knowledge and capacity. An index of the topics and presenters, with contact details, is in Appendix A along with links to recordings of all presentations. Participants also spent three hours in 14 working groups

\footnotetext{
Correspondence to: Geoff Warne, ILEP (e-mail: gwarne@ilepfederation.org)

${ }^{1}$ Towards zero leprosy: WHO global leprosy (Hansen's disease) strategy 2021-3030. New Delhi: World Health Organization, Regional Office for South-East Asia, 2021. https://www.who.int/publications/i/item/9789290228509.
} 
focusing on how ILEP can respond to the challenges. The working groups were structured to tackle the issue from varying perspectives involving combinations of high/low endemicity and high-/low-resource availability. This report summarises the findings of the groups.

\section{Sizing up the problem}

\section{TYPES OF KNOWLEDGE NEEDED}

The types of leprosy know-how required in countries at central, regional, district and community or grassroots level to get to zero leprosy are summarised in Table 1. The levels are inter-related: a lack of knowledge and awareness at one level also affects the other levels. As endemicity falls, case management and any complications are increasingly referred to specialist facilities, where the expertise needs to reside.

\section{PRIORITIES: THE MAIN GAPS IN LEPROSY KNOW-HOW}

There are knowledge gaps in all the categories listed in Table 1. These gaps are exacerbated in countries where leprosy-trained personnel are likely to be transferred to unrelated roles. Training in referral pathways is also seen as an important gap. Where there is ignorance of leprosy at PHC and community level, patients are usually referred to higher levels for treatment, but referral systems are beset by unsatisfactory communication and cooperation between different levels, often worsened by a combination of lack of interest and stigmatising attitudes among healthcare staff.

In lower-endemic settings, primary healthcare workers see so few cases that they may not retain even the basic knowledge to recognise leprosy and refer cases appropriately. A wideranging, composite approach is needed:

- Train people at community level, such as health workers, educators and community volunteers, to build capability in community-led case finding.

- Train associations or self-help groups of people affected by leprosy to help identify and refer potential cases to primary care facilities, navigate the health and social 'safety-net' system and use their expertise and personal experience to educate primary care workers on leprosy.

- Build the capacity of referral facilities. Specialist medical skills may be limited to only one, or very few, referral hospitals, and to a small number of expert staff who are approaching retirement without a new generation of skilled professionals to replace them.

- Integrate leprosy training into wider programmes, for example basic medical training and cross-NTD training.

- Consider the use of national or regional expert hubs.

Middle-level health managers have an important role. Training is often focused on the primary health and the upper echelons but not on the middle level. Here, a particular need is management and leadership training: the perspective to identify problems, find solutions, plan and analyse, and thus provide support to the levels above and below.

\section{EFFECTIVENESS AND SUSTAINABILITY OF CURRENT APPROACHES}

A remarkable array of approaches to skill and capacity development was identified. The following gained particular attention:

(a) Nationally recognised government or NGO training centres offer face-to-face theory and practical classes, useful (for example) for new programme managers and refresher 
Table 1. Types of leprosy knowledge needed

\section{Central or national level}

Epidemiological knowledge of the leprosy burden, including capability in mapping, data management, supply chain management and ongoing surveillance.

Sound understanding of leprosy, its impacts on the individual and community, and the interventions and continuum of care needed to achieve zero leprosy.

Integration or mainstreaming of leprosy with other health programmes.

Skills in messaging, mass communication and advocacy (especially in lower-endemic settings to ensure adequate resources for leprosy).

Understanding of capacity development and knowledge transfer.

Ability to maintain and update treatment guidelines.

Awareness of international trends in leprosy, including innovative tools and technology.

\section{First subnational level}

As for central/national level, with the addition of:

Expertise within regional referral services and training centres.

Programme management and supervisory skills.

Logistical skills especially supply of drugs, self-care kits and assistive devices.

Availability of expert technical support.

\section{District level, including within primary health centres}

Diagnosis and treatment, including skills in nerve function assessment and voluntary muscle testing, detecting hand, foot and eye involvement, because healthcare workers often look only for signs on the skin and miss symptoms of nerve damage.

Active case finding, contact tracing and post-exposure prophylaxis.

Diagnosis, management and (if necessary) referral of all forms of leprosy complications.

Disability prevention and both medical and socio-economic rehabilitation, accompanied by understanding of effective post-MDT surveillance.

Stigma reduction and mental health interventions including psychological first aid and counselling.

Working with DPOs and organisations of persons affected by leprosy, with an understanding of human rights.

Building and renewing capacity at all levels from community healthcare workers through to physicians, dermatologists, ophthalmologists and orthopaedic surgeons.

Advisory/advocacy services to facilitate social support and access to government schemes.

\section{Community or grassroots level}

Basic knowledge about leprosy and its manifestations.

How to suspect and refer cases.

Self-care techniques.

Methods of public information or awareness about leprosy.

How to counter or mitigate stigma and discrimination.

How to recognise and respond to poor mental wellbeing triggered by leprosy.

training. When staffed with qualified and experienced trainers, these are effective, but they are very expensive, reach only a small proportion of the workforce and present difficulties due to long course duration. Also, patients with symptoms are not always available.

(b) More localised workshops for health workers at various levels, including for nursing and medical college students, may be effective in practical learning and to reduce stigma, but there are challenges in making them sustainable where there is lack of resources.

(c) In-person, on-the-job training by doctors, supervisors etc. is effective as it involves hands-on practical training, but may not be sustainable in terms of time, and it can be challenging to find supervisors that are sufficiently trained to be able to teach adequately.

(d) E-learning targets workers at all levels, including volunteers, who visit the portal at their convenience to view or download training materials. Learning modules from WHO and 
other sources are readily available. But this training depends on internet connectivity and access to digital devices and lacks the hands-on experience of physical examination.

(e) Regional WHO offices offer periodic training, for example on newly published guidelines, but these workshops are typically narrowly focused and are not ongoing.

(f) Leprosy capacity building can be integrated into other well-resourced disease training programmes, thus providing leprosy training for health workers who would not attend a specifically leprosy course. Examples included TB, integrated skin diseases, and footcare training. Sometimes, attending courses on these topics is the first time the trainee hears about leprosy. This approach is effective and sustainable in building capacity.

(g) Organisations of persons affected by leprosy in some countries offer training in human rights-based approaches, advocacy, messaging and community-based development. This training is effective in capacity development and empowers persons affected. It is usually voluntary and ad hoc: investment would be needed to make it more structured and sustainable.

(h) At community level, media centres, posters, songs and street plays sharing simple facts on leprosy are effective in raising awareness, including among community and religious leaders. The use of small adverts and animations in local languages has also been appreciated by rural communities.

(i) Digital tools like SkinApp ${ }^{2}$ and $\mathrm{Siilo}^{3}$ (for groups to communicate together after training) have been a valuable adjunct to other forms of training. As languages are added, SkinApp is becoming an increasingly important resource.

\section{ALTERNATIVE, MORE EFFECTIVE AND SUSTAINABLE APPROACHES}

Blended courses, combining online or app-based learning sessions with offline practical training in hubs, are found to be better than e-learning alone. Listening, online viewing and reading lead to knowledge, whereas practical experience with patients leads to acquisition of both knowledge and skills. Some skills are considered very difficult to acquire other than through in-person instruction. Nevertheless, watching pre-recorded video on certain practical topics could help reduce the duration of training, and learners may be able to demonstrate skill acquisition by video to an expert observer. In high-resource settings virtual reality could be trialled. There would be value in mapping both the subject-matter for which in-person training is essential and the places in which that training can be made available.

Other ideas included:

- The use of simple, cost-effective, existing digital communication platforms to confirm diagnosis (such as WhatsApp groups with technical experts) and social media to share experiences/information and raise awareness.

- Mentoring along with self-practice and problem-based learning.

- Inclusion of leprosy in medical education and curricula. The material should cover not just diagnosis and case management but also social exclusion and mental well-being.

- Especially in low-endemic settings, having experts available digitally to consult regarding suspect cases and complication management.

- Learning from the virtual training experience of universities and organisations working on specialised issues, such as advocacy and human rights.

\footnotetext{
${ }^{2}$ Stepping up leprosy prevention with the SkinApp | NLR (nlrinternational.org).

${ }^{3}$ Siilo | Free Secure Medical Messaging App.
} 


\section{Capacity development: what people need to know}

THE CONTENT OF AN EFFECTIVE CAPACITY DEVELOPMENT PROGRAMME

Participants devised a detailed curriculum outline, suited to high and low-endemic contexts, for the different target groups: community health workers at the PHC level, secondary care facilities and supervisors, tertiary care facilities, traditional healers, the community, people affected by leprosy, students and young people, and managers.

\section{CONTENT: WORLDWIDE OR COUNTRY-SPECIFIC?}

The basic knowledge is the same worldwide, but some aspects need to be tailored to the social and cultural norms of a particular region or country and to other contextual factors, so that persons being trained can relate easily to it. Examples of contextual factors include integration of leprosy with other health programmes, different systems, different definitions, different disability support and social entitlements, different regimens, supply chain differences, level of stigma, existence of active groups of persons affected by leprosy, and government-specified curricula. Pictorial material needs to be adapted to common skin tones and other presentation features in a target country.

AVAILABILITY OF CONTENT AT THE RIGHT QUALITY, ACADEMIC LEVEL, LANGUAGE ETC

A great deal of leprosy teaching and learning material is available, in a range of formats, with WHO, ILEP, governments, and sometimes with organisations of persons affected by leprosy (such as Morhan, Brazil). But materials are often not kept updated and there are significant gaps, so it needs to be collated, revised and upgraded and then translated. Moreover, material may not suit the target audience and therefore, to be effective, it needs to be adapted culturally and pitched at the right academic levels and with attention to higher and lower endemicity contexts. There are some intriguing approaches to assessing training needs: in Brazil, for example, a partnership with Google looks at commonly searched terms on leprosy so as to identify information gaps.

\section{WHAT ILEP CAN OFFER}

ILEP is rich in clinical expertise and experience in training of healthcare providers, and is able to pull together experts not only in the full range of leprosy-related disciplines but also in other NTDs, disability, mental health etc. ILEP, through its networks, could be the leading voice to improve stakeholder coordination between and within countries and to merge the necessary funds from various sources. ILEP should make an inventory of all teaching materials that are available and have proven effective, facilitate consultations to identify gaps and training needs, and both advocate and work with governments to build or update content for leprosy training materials in a range of languages, styles and levels within the health system to achieve a broader reach. Conversely, ILEP should also work towards the integration of leprosy-specific material into learning materials for specialists working in ophthalmology, disability management, management of foot ulcers, peripheral nerve damage and other relevant disciplines.

\section{Learning design}

STAKEHOLDERS IN DECISIONS ON THE DESIGN OF LEARNING PROGRAMMES

As many stakeholders as possible should be included in decision-making about the way a learning programme is designed, to ensure that the learning is successfully implemented 
Table 2. Stakeholders and contributors in the design of leprosy learning programmes

\section{Governments}

Demonstrate political commitment by taking the lead in developing policies.

Convene stakeholders in health, social services and education, and persons affected by leprosy, to discuss the best approaches to learning programmes in leprosy, and then appoint a suitable taskforce and accredit the developed programme for dissemination and implementation.

Provide the bigger picture of which services are available, how leprosy training should best be integrated into other curricula, and what digital and other approaches are being used in learning design in other disciplines.
\end{abstract}

\title{
NGOs including ILEP
}

Influence the government to ensure that leprosy is included in capacity development thinking at all levels. Facilitate stakeholder engagement.

Provide internal and external technical expertise on programme design from their experience including (particularly) at the grassroots. NGOs working in leprosy will often be the expert training providers, especially with the practical components of training, and be able to provide ongoing mentoring.

\section{Subject-matter experts and professional bodies}

Includes not just scientists but people who work at the grassroots, who are experts in implementing leprosy programmes and can advise on what learning approaches are likely to be workable at community level.

Contribute to decisions on programme design, especially how to implement a suitable blend between theory and practice in learning.

Validate the educational packages produced.

\section{Education experts}

Verify that the training content is appropriately focused.

Select the most effective teaching methodology for the different levels.

Ensure that government is aware of digital training programmes and good practice.

Help prepare assessment tools.

\section{Technology companies}

Provide information from an early stage on what functionality is available to a training platform, what is possible digitally in a given context and what are the implications for course design.

\section{World Health Organization}

Contribute a degree of uniformity to education material.

Endorse learning programme design.

Make sure that developed programmes are widely disseminated between countries.

Influence a political commitment to leprosy training in the context of the WHO NTDs roadmap 2021-2030. ${ }^{a}$

Use its global overview to help to minimise unnecessary duplication in training development.

\section{Organisations of persons affected by leprosy}

Participate in programme design, as in all other aspects of capacity development.

Ensure that issues of importance to people with lived experience of leprosy are included and that their stories and experiences have a place in the training programme.

Contribute as trainers and peer educators.

Advocate to ensure leprosy training is not overlooked, and monitor its implementation.

\section{Researchers}

Build evidence as to which approaches and materials are most effective.

\footnotetext{
${ }^{a}$ Ending the neglect to attain the Sustainable Development Goals: a road map for neglected tropical diseases 20212030. Geneva: World Health Organization, 2020. https://www.who.int/publications/i/item/9789240010352.
}

and adapted to the needs. Key stakeholders and contributors include governments, NGOs including ILEP, subject-matter experts and professional bodies, education experts, technology companies, World Health Organization, organisations of persons affected by leprosy, and researchers. Their roles are summarised in Table 2. 


\section{LEARNING PROGRAMME DESIGN: WORLDWIDE OR COUNTRY-SPECIFIC?}

While some principles could be the same globally, successful learning designs, approaches and materials need to take into consideration the country context, endemicity, resource availability, IT infrastructure, and literacy and educational attainment at different levels of the workforce. The solution could be a generic framework and a universally applicable package of resources (including e-learning, apps, online readings, videos, animations, written summaries etc) designed with the explicit intention of country/context-specific adaptation.

\section{LEPROSY-SPECIFIC VERSUS INTEGRATED LEARNING DESIGN}

Although in high-endemic settings there may be a stronger case for leprosy-specific training, most participants agreed that an integrated approach, with other relevant NTDs for example, is more sustainable and reduces stigmatisation related to leprosy. Integrated approaches are especially favoured at or near the frontline where health workers see people with every kind of disease and need a broad knowledge. Integration also helps to draw people who would not have chosen a specifically leprosy programme but may attend a training programme that includes leprosy within a range of NTDs or other diseases. For more complicated and specialist services, it is important to maintain specialised leprosy expertise, so in these situations a leprosy-specific approach is more justified and reduces the risk that specialist knowledge and practice may get lost.

\section{PEDAGOGICAL (TEACHING AND LEARNING) STRATEGIES}

Different target groups require different pedagogical strategies for the course, the numbers of trainees, the objective and content of the training, and the educational level of the participants. As noted earlier, blended learning approaches, combining either classroom teaching or elearning with hands-on clinical experience, are considered the most effective. Other factors that need to be taken into account include the training of trainers, the availability of people for mentoring, the availability of specialised leprosy facilities for advanced skills, and accreditation or course completion certificates from national or international bodies.

Education experts should be commissioned to design the best teaching strategies. Recommended practices included:

- Interaction: question and answer, problem-based learning through case studies, web-based journal clubs and case conferences, quizzes, role plays. The ECHO Institute's case-based learning method gained special mention. ${ }^{4}$

- Communities of practice that support maintenance of skills through regular exchange. People join when it is relevant for their work.

- App-based learning (the SkinApp, for example) for both clinical decision-making and learning. Trainees practise recognising common skin diseases including leprosy.

- E-learning modules and webinars for self-study or group study. Learners can revisit what was learnt and to absorb the material at their own pace.

- Highly visual, pictorial, practical approaches and simple layman's language for health workers with lower literacy or educational attainment. Interest may be lost if there is too much information.

- Flexibility so that what is provided can be accessed according to how people want to learn.

${ }^{4}$ https://hsc.unm.edu/echo/echos-impact. 
- Use of animations and puppets, especially to address difficult, controversial, or taboo topics: the animated character or puppet cannot be blamed for what it says.

- Learning through interaction with leprosy champions and people affected by leprosy.

\section{ASSESSMENT OF LEARNING OR SKILLS DEVELOPMENT}

Assessment is crucial, because the purpose of training is to get impact. The knowledge level should be tested before and after training, using skill-based objective scoring, to evaluate the effect of the training. Assessments should cover both theory and practice, so trained leprosy supervisors would be needed to verify that the skills had been learnt. Further posttests after one to two years would provide assurance that the knowledge is being retained, and these could be in the context of follow-up workshops coupled with buddying/networking and mentoring/coaching. Follow-up training of this kind emphasises the importance of continuous learning. Objective measures such as numbers of contacts traced, more timely diagnoses and lower disability, and input from new leprosy patients about their experience in diagnosis, might offer further assurance that skills had been learnt and retained.

\section{WHAT ILEP CAN OFFER}

ILEP can bring local and international experts together, influence the government in the design of capacity development programmes and national training policies, and participate directly in training. In addition, ILEP's experience in the field means it can often supply pictures and case studies, and it can encourage the participation of persons affected by leprosy in learning design and programme implementation. ILEP is well placed to supervise the running of a pilot of training materials, help to design and support assessment processes, and share what is learnt from various countries. Participants looked to ILEP to have a vision for active learning, which has an empowering effect on the individual.

\section{Mode of delivery}

\section{EFFECTIVE DIGITAL LEARNING APPROACHES}

As has already been noted, blended approaches are expected to have the best results: trainees need to complete both knowledge and skill assessment. Some e-learning methods are already being used, and it makes sense to gradually formalise what is already in place and learn from that experience before pushing further.

In higher-resource settings, a person can access a self-directed e-learning course-podcast, online module, clinical app, webinar-from anywhere. Offline there would need to be locations for practical skill acquisition, though it had already been noted that this could be challenging in lower-endemic settings. Considering how important it is to see many cases of leprosy before a person begins to thoroughly understand the disease, virtual reality could, potentially, enable a person to gain clinical knowledge or experience or even to 'feel' leprosy without having to attend a clinic in person. Simulation mannequins are another option to consider.

In lower-resource settings, learning can be adapted to the platforms that people already use (WhatsApp, SMS, YouTube etc). Other options are short audio and/or video messages, interactive learning through low-bandwidth apps, and identifying learning platforms that work well with low bandwidth. Experience and comfort in using technology may be an issue for some.

E-learning is not only for the health workforce. Self-help groups and associations of persons affected by leprosy may be able to use core materials and use their experience and group 
dialogue for in-person or virtual interactive learning among themselves. Members can then share their knowledge and training resources with primary health workers.

\section{FUTURE ROLE OF TRADITIONAL APPROACHES TO TRAINING}

New digital approaches to training need to be embedded and their effectiveness evaluated. Where feasible, traditional approaches to learning, such as training centres or institutes, should co-exist for the time being, while ILEP and partners explore the right balance of e-learning and face-to-face training for various cadres of trainee. Although face-to-face training may enhance the learning achieved through digital platforms, its main focus needs to be on the practical aspects, and it may never be replaced for skills acquisition in, for example, reconstructive surgery, post-surgical management and nerve examination. Training centres play a valuable role as a repository of expert knowledge and as a hub for researchers.

In lower-resource settings, especially in lower-endemic countries, maintaining leprosy training centres may not be feasible due to cost. These are likely to be phased out in favour of more innovative approaches, though solutions are still needed for those elements of training that need hands-on learning. Communities of practice, other group interactions, and coaches or mentors accessed by mobile phone may all have a part to play in lower-endemic, lowerresource settings.

\section{OBSTACLES TO SUCCESSFUL DIGITALLY BASED LEARNING}

Even in higher-resource countries, tech literacy, limited bandwidth and access to relevant hardware can be major issues. There are useful examples from other settings as to ways these issues can be overcome,${ }^{5}$ though discernment is needed: some technologies that have appeared innovative did not have a long-term future. In lower-resource countries, trainees may have access only to a smartphone or not even that. Computer hardware investment could be needed not just for teaching but also for the trainee. Where possible, the learning content should be able to be downloaded and later accessed offline, so that trainees can learn at their own speed.

Motivation, participation and engagement may be lower in digital training. Systems need to be developed to accurately monitor the training programmes and ensure that people are suitably accredited when they finish the course. Use of eminent international presenters may help make the training more attractive. Trainees unaccustomed to e-learning will need to adapt to a different way of learning. They can be supported and motivated by adaptation of materials to local contexts and languages; provision of technical support; and incentives for use of the platforms by provision of completion certificates.

\section{ENSURING SUSTAINABILITY}

Governments have the mandate to require health workers to undergo training and to make certain training mandatory for some senior positions. Demonstrating the effectiveness of this training through monitoring and feedback systems encourages governments to continue to support it. Although some cadres of health workers are routinely moved or transferred, sustainability is boosted where health workers, especially at the front line, remain in their communities. Similarly, governments and NGOs can ensure that persons with know-how understand that knowledge sharing and mentoring are part of the job. There should be a clear

\footnotetext{
${ }^{5}$ For example, use of 'Zuba boxes', solar powered internet labs (https://www.computeraid.org/work-projects/proj ects/our-solar-learning-labs and https://en.wikipedia.org/wiki/ZubaBox).
} 
commitment by government and NGOs to welcome trainees to specialist referral centres for the hands-on element of the training programme.

Sustainability is also a financial issue. Governments should be lobbied to include leprosy in their training programmes and budgets. ILEP or its partners can help demonstrate how digital methods can reduce costs, and may lobby for technology companies to discount their services to develop digital training programmes. Leprosy skill development should be integrated within other health and education programmes, into other diseases or specialities, and into existing elearning and other training platforms. Accessing free or discounted training from sites like http://www.openwho.org and http://www.oercommons.org/ can ease strain on the training budget.

The training role of organisations of persons affected by leprosy has been referred to previously. Those with access to training materials and resources can contribute to sustainability by continuing to train community members and health workers. They might need support from NGOs to participate in lower-resource areas.

\section{WHAT ILEP CAN OFFER}

In terms of innovative delivery of capacity development, ILEP's main roles are in-country. While lobbying government to budget the necessary funds to sustain leprosy expertise at national and subnational levels, ILEP should be prepared to complement with its own resources, training expertise and technical support. Through its connections ILEP can also map other resources available in-country, including from partners in the NTD world and beyond. Because undergraduate training in leprosy is often substandard and interest is low, ILEP's lobbying should extend to ensuring incorporation of leprosy within medical and nursing students' curricula.

ILEP can support the hands-on aspects of training especially where it is able to influence referral hospitals. This will enable students in medical, nursing and other health professions to be posted to leprosy referral centres for skill-based mentorship by experts. As technical consultant, ILEP can facilitate the development of an inventory of innovative delivery methods that produces an overview of what works best and in which context. It can then share the information, and work with all training providers to ensure that the best methods are used nationally.

Internationally, ILEP can contribute to development and research on innovative learning and capacity development activities. Participants welcomed the concept of an international leprosy academy, and there was enthusiasm for the prospect of a common action plan for ILEP members to ensure collaboration and partnerships in capacity development.

\section{Additional suggestions}

OPERATIONAL RESEARCH INTO FACETS OF CAPACITY DEVELOPMENT

The following topics were proposed for operational research:

- Indicators providing evidence of real-world impact from leprosy training programmes.

- Feasibility of use of virtual reality in leprosy skills development.

- Impact and sustainability of digital versus traditional learning approaches in various settings. This includes further exploration of the use of the ECHO Institute model in leprosy.

- Benefits of integrated training, which other diseases leprosy training is best integrated with, and to what extent integration should be universal or differ according to the context.

- Extent to which internationally developed content is suitable for different countries. 
- Efficacy studies before standardising learning content, design and mode of delivery.

- Effective models for engagement of persons affected by leprosy in capacity development within the health service.

- Understanding the different needs and motivations of capacity development target groups.

This research can be time-consuming. Some participants preferred implementation research in which innovative approaches are piloted, tested, adapted, scaled and then shared.

\section{OTHER INNOVATIVE APPROACHES}

Other innovative ideas, not previously covered, included:

- Messaging aimed at building the next generation of leprosy experts and motivating health professionals to commit to leprosy work. Advocacy could emphasise (a) the legacy they could have, defeating leprosy in their lifetimes; and (b) the human rights perspective, showing leprosy as more than a medical issue.

- Follow-up of training: investment in support networks and ongoing professional development to ensure that knowledge remains.

- A leprosy resource call centre from where knowledge can be accessed by phone.

- Developing channels dedicated to leprosy training that are attractive and easy to use for the target group, including podcasts, blog articles, videos, audios etc.

- The participation of people affected by leprosy is crucial: there needs to be a deliberate journey from informing to consulting to involving to collaborating to empowering.

- Innovative integration, recognising the challenge of retaining interest in leprosy alone.

- Innovation can include combining and modifying existing techniques, as well as entirely new.

WHAT ILEP CAN OFFER

Other suggestions, not previously covered, included:

- Form a working group to take the subject forward in a coordinated way, involving ILEP members and other organisations interested in capacity development in this sector.

- Develop an evidence-based business case for investing in leprosy capacity development, including the cost of not investing. This may help to influence governments and other funders.

- Position Infolep 6 as a comprehensive training resource.

- Map potential solutions and innovative approaches and enable synergies amongst ILEP members in terms of building leprosy know-how and capacity.

- Identify and build a network of potential experts and trainers across all ILEP members. The network would become the go-to resource for teaching, hands-on training, mentoring and as a pool of experts for referral of rare cases (especially in low-endemic settings).

\section{Conclusion and next steps}

The high level of engagement by conference participants produced a rich harvest of innovative thinking. For ILEP and its member associations, some of the most significant input is in the various sections entitled 'What ILEP can offer'. ILEP is currently debating its strategy

${ }^{6}$ https://www.leprosy-information.org. 
for the next five years, in which capacity development is certain to be a key focal area. One way in which this is already being demonstrated is in the workplan for the ILEP Technical Commission (ITC). The workplan includes eleven capacity development topics for which work products are either totally lacking, and thus need to be designed and developed, or need major modification. A dedicated subgroup is being established for this task.

\section{Acknowledgements}

The authors wish to thank all the conference presenters and the working group facilitators and rapporteurs. Sincere appreciation to Sunil Anand, Sundeep Chaitanya, Jannine Ebenso, Paul Watson, Christine Fenenga and especially Sian Arulanantham for their support, advice and knowledgeable input as members of the conference planning team.

\section{Appendix A}

Table A1. Presentations on innovative approaches to the challenges around leprosy knowledge and capacity

\begin{tabular}{|c|c|c|c|}
\hline Title & Presenter & Organisation & Contact \\
\hline $\begin{array}{l}\text { Adapting e-learning } \\
\text { approaches for leprosy } \\
\text { capacity development }\end{array}$ & Sian Arulanantham & $\begin{array}{l}\text { The Leprosy } \\
\text { Mission England } \\
\text { and Wales }\end{array}$ & siana@tlmew.org.uk \\
\hline $\begin{array}{l}\text { Coordinated approach to } \\
\text { building disease know-how }\end{array}$ & Claire Jennings & TEPHINET & cjennings@tephinet.org \\
\hline $\begin{array}{l}\text { Democratizing health } \\
\text { knowledge }\end{array}$ & Sanjeev Arora & $\mathrm{ECHO}$ & SArora@salud.unm.edu \\
\hline WHO e-learning animation & Pemmaraju Rao & $\begin{array}{l}\text { WHO Global } \\
\text { Leprosy } \\
\text { Programme }\end{array}$ & pemmarajuv@who.int \\
\hline Role of people's & Paula Brandäo & ILEP Advisory & pbrandaofenf@gmail.com \\
\hline $\begin{array}{l}\text { organizations in maintaining } \\
\text { leprosy know-how }\end{array}$ & Mathias Duck & Panel & mathiasduck@hotmail.com \\
\hline $\begin{array}{l}\text { Telemedicine for skin } \\
\text { conditions }\end{array}$ & Shimelis Nigusse & ALERT Ethiopia & shimelis321@gmail.com \\
\hline $\begin{array}{l}\text { New approaches in leprosy } \\
\text { training including virtual } \\
\text { classroom }\end{array}$ & David Prakash & Karigiri & deenaanddavid@gmail.com \\
\hline $\begin{array}{l}\text { E-Learning program for skin } \\
\text { NTDs }\end{array}$ & Christian Johnson & FRF & rochchristianjohnson@gmail.com \\
\hline $\begin{array}{l}\text { Building capacity is a } \\
\text { necessity }\end{array}$ & Liesbeth Mieras & $\begin{array}{l}\text { NLR } \\
\text { International }\end{array}$ & L.Mieras@nlrinternational.org \\
\hline Blended learning & $\begin{array}{l}\text { Fransje van der } \\
\text { Waals }\end{array}$ & Health(e)Foundation & vanderwaals@biomed.nl \\
\hline
\end{tabular}

Recordings can be viewed as follows:

Sessions 1 and 2: https://www.dropbox.com/sh/cm0xelaijjzmw1e/AADLChPo-KQR2q2aE3r-SyXma?dl=0. Sessions 3-5: https://www.dropbox.com/sh/oj0p7jg3kxl0uen/AAAWgt4qgCxbIbeBmuod0raZa?dl=0.

Sessions 6-8: https://www.dropbox.com/sh/qnwhynyut8smuoo/AACWGcuaHAOc_p604x8BMSVEa?dl=0. Sessions 9-10: https://www.dropbox.com/sh/3uio0mk91j5fkje/AACJAmeTOsDt9q8E5Sv_VaKfa?dl=0. 